\title{
Pore Characteristics and Hydrothermal Stability of Mesoporous Silica: Role of Oleic Acid
}

\author{
Junhyun Choi, ${ }^{1}$ Yosep Han, ${ }^{1}$ Soyeon Park, ${ }^{1}$ Jayhyun Park, ${ }^{2}$ and Hyunjung Kim ${ }^{1}$ \\ ${ }^{1}$ Department of Mineral Resources and Energy Engineering, Chonbuk National University, 567 Baekje-daero, \\ Deokjin-gu, Jeonju, Jeonbuk 561-756, Republic of Korea \\ ${ }^{2}$ RઐD Team, Institute of Mine Reclamation Corporation, Coal Center, 30 Chungjin-dong, Jongno-gu, Seoul 110-727, Republic of Korea
}

Correspondence should be addressed to Jayhyun Park; jayhp@mireco.or.kr and Hyunjung Kim; kshjkim@jbnu.ac.kr

Received 27 April 2014; Accepted 23 June 2014; Published 6 July 2014

Academic Editor: Donglu Shi

Copyright (C) 2014 Junhyun Choi et al. This is an open access article distributed under the Creative Commons Attribution License, which permits unrestricted use, distribution, and reproduction in any medium, provided the original work is properly cited.

\begin{abstract}
Silicate mesoporous materials were synthesized with nonionic surfactant and their surfaces were modified by oleic acid adsorption. Infrared spectrometer, nitrogen adsorption-desorption isotherm, scanning electron microscopy, and thermogravimetric analyses were used to investigate the structure of oleic acid modified mesoporous material. The effects of heat treatment at various temperatures on oleic acid modified materials were also studied. Oleic acids on silica surfaces were found to be bonded chemically and/or physically and be capable of enduring up to $180^{\circ} \mathrm{C}$. The adsorbed oleic acid improved the hydrothermal stability of mesoporous silica and assisted mesopore structure to grow more in hydrothermal treatment process by preventing the approach of water.
\end{abstract}

\section{Introduction}

The mesoporous silica, which has the characteristics of high specific surface area, ordered pore structure, and large pore volume, has been paid attention owing to potential applications as a catalyst support, adsorbent for environmental material [1]. However, the practical applications of the mesoporous silica have been limited by the poor stability of high-surface amorphous silica to moist atmosphere. The stability of mesoporous silica is probably affected by the hydrolysis of silica surface with water molecule $[2,3]$. A lot of researchers have proposed the silylation of silica surface and the zeolitization of the silica wall as the ways to improve the hydrothermal stability of the silica material [4-7]. Zeolitization of the mesoporous silica wall could be expected to get good hydrothermal stability, like zeolite materials, to the mesoporous silica. Many researchers tried to study this synthesis but the case of successful syntheses is not reported yet. In most cases, zeolite and mesoporous materials were synthesized separately $[8,9]$. Though it was reported that some zeolites with mesopore were synthesized, a surfactant as a structural direct agent was not used in their synthesis process [10]. In contrast, the silylation of mesoporous silica surface is the practical method to improve hydrothermal stability. The silylation process could stabilize the silica surfaces by changing hydrophilic property into hydrophobic property that interrupts the access of water molecule to silica surface. Moreover, silylated silica surfaces have found several applications in chemistry (adsorbent for chromatography, catalyst), biochemistry (enzyme immobilization), and electronic (low dielectric material) [1113]. However, there are some methods modifying hydrophilic property of silica surface into hydrophobic property as well as the silylation $[14,15]$. One of generally known methods is the surface modification with oleic acid. The oleic acid adsorption is an alternative method to obtain hydrophobic property of silica surfaces, except the case of being used in high temperature condition. The adsorption properties of silica with oleic acid used as a surface modification agent to improve disperse stability in organic solvent and oil have been studied because of their great practical importance and the widespread industrial use of silica materials as catalysts and adsorbents [16]. Therefore, if silica surfaces are capable of 
being stabilized hydrothermally by silylation process, it may be also stabilized by oleic acid modification. In the present work, oleic acid modified mesoporous silica particles were synthesized using surface modification method in organic solvent, and the thermal stability and hydrothermal stability of surface modified material were investigated.

\section{Materials and Methods}

2.1. Synthesis of Mesoporous Silica Modified by Oleic Acid. In a typical preparation, $4 \mathrm{~g}$ of Pluronic P123 (Aldrich) was dissolved in $120 \mathrm{~g}$ of $2 \mathrm{M} \mathrm{HCl}$ solution with stirring at $35^{\circ} \mathrm{C}$ [17]. Then, $46 \mathrm{~g}$ of silicate solution made by dissolving solid state amorphous silica (purity 99\%, Dongyang Chem. Co.) in $1 \mathrm{M} \mathrm{NaOH}$ solution was added into that solution at $35^{\circ} \mathrm{C}$ [18]. The mixture was aged at $90^{\circ} \mathrm{C}$ for $20 \mathrm{~h}$ without stirring. The precipitated solid product was recovered, washed, and dried at $60^{\circ} \mathrm{C}$. In order to remove the surfactant, $3 \mathrm{~g}$ of solid was treated with $500 \mathrm{~mL}$ of an ethanol at room temperature for $16 \mathrm{~h}$, then filtered, washed, and dried. Oleic acid adsorption was performed by stirring $2.9 \mathrm{~g}$ of solid in $300 \mathrm{~mL}$ of $0.1 \mathrm{M}$ oleic acid (Shinyo) in $\mathrm{n}$-hexane (Samchun) at room temperature overnight. The products were filtered, washed with deionized water, dried, and heat treated at $80^{\circ} \mathrm{C}, 120^{\circ} \mathrm{C}$, $180^{\circ} \mathrm{C}$, and $240^{\circ} \mathrm{C}$, respectively, for $1 \mathrm{~h}$.

2.2. Stability Test. To test the hydrothermal stability, materials were heated at $105^{\circ} \mathrm{C}$ and refluxed in water. For the hydrothermal treatment, $0.5 \mathrm{~g}$ of samples was dispersed in $100 \mathrm{~mL}$ of deionized water, and the mixed solutions were heated in an autoclave without stirring at $105^{\circ} \mathrm{C}$ for $6 \mathrm{~h}$. For water treatment, samples were refluxed at room temperature for $24 \mathrm{~h}$. The hydrothermal treated and water treated materials were recovered by filtration and dried.

2.3. Characterization. The images of the IR spectra were determined in transmission mode using potassium bromide (KBr) based disk pellets prepared by mixing $\mathrm{KBr}$ powder with sample powder. The spectra were measured in the range $4000 \mathrm{~cm}^{-1}-400 \mathrm{~cm}^{-1}$, with a resolution of $2 \mathrm{~cm}^{-1}$, using a Fourier transform infrared spectrometer (Nicolet, Omnic 405 Model). Thermogravimetric analyses of the samples were performed with a TA Instruments SDT2960. The sample powders were heated in flowing air from ambient temperature to $400^{\circ} \mathrm{C}$ at a rate of $5^{\circ} \mathrm{C} \mathrm{min}^{-1}$. Surface and shape of the mesoporous samples were examined with a field-emission scanning electron microscope (JSM-6330F, JEOL). Transmission electron microscopy (TEM) observations were conducted with a JEOL-2100F microscope operated at $200 \mathrm{kV}$. The samples for TEM measurements were suspended in ethanol and dropped onto holey carbon films that were supported on $\mathrm{Cu}$ grids for imaging. Specific surface area was calculated from the data obtained from partial relative pressure that ranged between 0.05 and 0.30 using the Brunauer-Emmett-Teller (BET) method [19]. Pore volume and pore size distributions were determined from the amount of nitrogen adsorbed at a relative pressure of 0.99 and by the Barrett-Joyner-Halenda (BJH) method [20].

\section{Results and Discussion}

3.1. Effects of Oleic Acid on the Pore Characteristics of Mesoporous Silica. All materials were prepared at a very low $\mathrm{pH}(<1)$. The aggregates of synthesized amorphous mesoporous materials are like long fabric shape (Figure 1(a)). The surface is apparently smooth and seems to act like cement (Figure 1(b)). After oleic acid modification, the aggregates had no specific shape (Figure 1(c)) and the surfaces are entirely flat and small spherical shape is mixed on the flat surface (Figure $1(\mathrm{~d})$ ). This indicates that oleic acid helps improve the dispersity of silica particles in organic solvent that was used in the pretreatment of SEM.

The nitrogen adsorption-desorption isotherms of synthesized materials are shown in Figure 2. The adsorptiondesorption isotherms belong to type IV of the IUPAC classification, which is a characteristic of mesoporous material. The one steep slope at $0.7-0.8$ of $P / P_{0}$ would be shown on porous adsorbents possessing uniform mesopores of approximately 9-11 nm. They also showed a type A hysteresis loop of de Boer located at relatively high pressure than that of MCM41 material. It is known that this kind of hysteresis loop is due to cylindrical pores open at both ends [21]. This hysteresis is caused by condensation and evaporation of a hemispherical meniscus produced in cylindrical pore. The surface modification process by oleic acid brought about a decrease in the nitrogen adsorption capacity, reflecting a decrease in the specific surface areas and pore volumes of the samples.

The specific surface area and pore volume of untreated original sample were $480 \mathrm{~m}^{2} / \mathrm{g}$ and $0.83 \mathrm{~cm}^{3} / \mathrm{g}$, respectively, and decreased to $270-310 \mathrm{~m}^{2} / \mathrm{g}$ and $0.6-0.63 \mathrm{~cm}^{3} / \mathrm{g}$ for oleic acid modified silica (Table 1 ). The reduction in pore volume and specific surface area by simple coating of oleic acid appears to be related to the structure of mesoporous substrate since oleic acid may fill micropores and mesopores. The oleic acid coating on silica surface resulted in the decrease of micropore volume observed from isotherms at low relative pressure $\left(P / P_{0}<0.3\right)$ (Figure 2$)$. However, in the case of the samples treated at $240^{\circ} \mathrm{C}$, specific surface area and pore volume were observed to suddenly increase (Table 1), and the pore structure (i.e., micropore and mesopore volumes) was also better developed than those for untreated original samples (Figure 2).

Figure 3 shows the pore size distributions determined by applying the $\mathrm{BJH}$ model to adsorption branches of the nitrogen physisorption isotherms in Figure 2. The results showed that the oleic acid modification caused not only a decrease in the adsorption capacity of samples, but also a decrease in mesopore size (Figure 3 ). The pore diameter of oleic acid modified samples decreased about $2 \mathrm{~nm}$ with respect to the nonmodified original sample.

\subsection{Effects of Oleic Acid on the Surface Characteristics of Meso-} porous Silica. The surface groups of mesoporous silica before and after oleic acid modification were characterized by FTIR. The spectra for the nonmodified sample and the oleic acid treated samples with thermal treatment at different temperatures are shown in Figure 4. 
TABLE 1: Specific surface area and pore volume of nonmodified sample and oleic acid (OA) modified samples with heat treatment at various temperatures before and after hydrothermal and water treatment.

\begin{tabular}{|c|c|c|c|c|c|c|}
\hline \multirow{2}{*}{$\begin{array}{l}\text { Heat treatment } \\
\text { condition }\end{array}$} & \multicolumn{2}{|c|}{ Before treatment } & \multicolumn{2}{|c|}{ Hydrothermall treatment at $105^{\circ} \mathrm{C}$} & \multicolumn{2}{|c|}{ Water treatment at $25^{\circ} \mathrm{C}$} \\
\hline & $\begin{array}{l}\text { Specific surface area } \\
\left(\mathrm{m}^{2} / \mathrm{g}\right)\end{array}$ & $\begin{array}{l}\text { Pore volume } \\
\left(\mathrm{cm}^{3} / \mathrm{g}\right)\end{array}$ & $\begin{array}{l}\text { Specific surface area } \\
\left(\mathrm{m}^{2} / \mathrm{g}\right)\end{array}$ & $\begin{array}{l}\text { Pore volume } \\
\left(\mathrm{cm}^{3} / \mathrm{g}\right)\end{array}$ & $\begin{array}{l}\text { Specific surface area } \\
\left(\mathrm{m}^{2} / \mathrm{g}\right)\end{array}$ & $\begin{array}{l}\text { Pore volume } \\
\left(\mathrm{cm}^{3} / \mathrm{g}\right)\end{array}$ \\
\hline Nonmodified & 480 & 0.83 & 332 & 0.51 & 397 & 0.64 \\
\hline Heat treated at $80^{\circ} \mathrm{C}$ & 272 & 0.6 & 604 & 1.09 & 518 & 0.83 \\
\hline Heat treated at $120^{\circ} \mathrm{C}$ & 314 & 0.62 & 661 & 1.07 & 521 & 0.79 \\
\hline Heat treated at $180^{\circ} \mathrm{C}$ & 284 & 0.63 & 512 & 0.86 & 390 & 0.64 \\
\hline Heat treated at $240^{\circ} \mathrm{C}$ & 674 & 0.99 & 415 & 0.88 & 680 & 0.95 \\
\hline
\end{tabular}

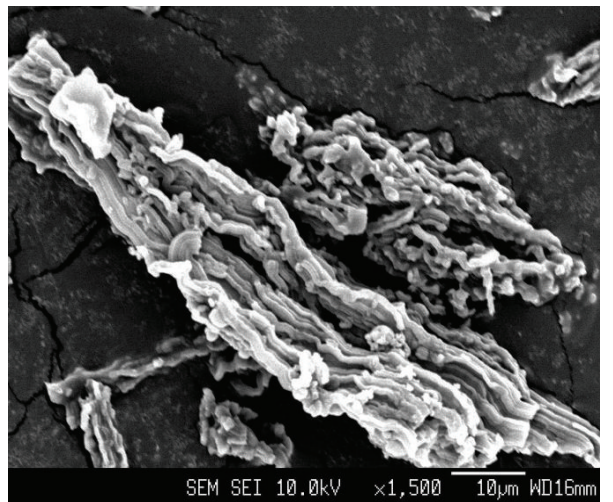

(a)

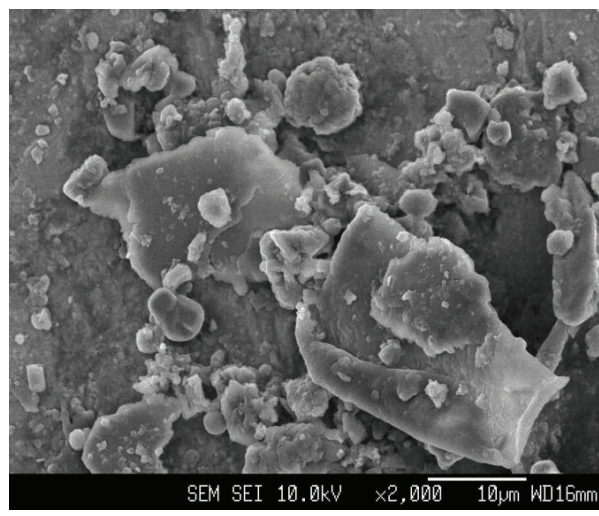

(c)

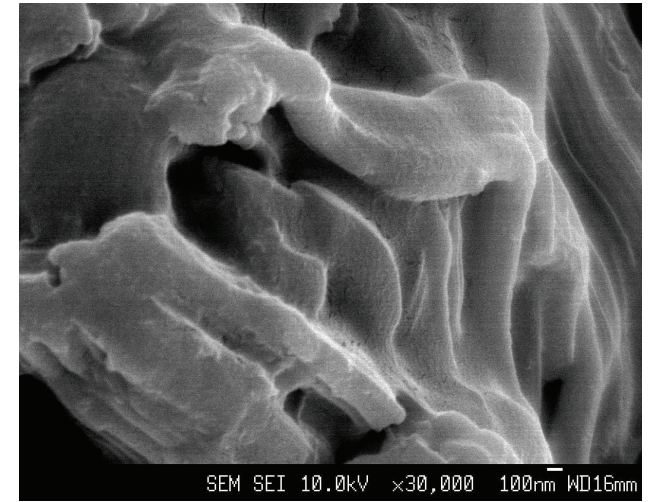

(b)

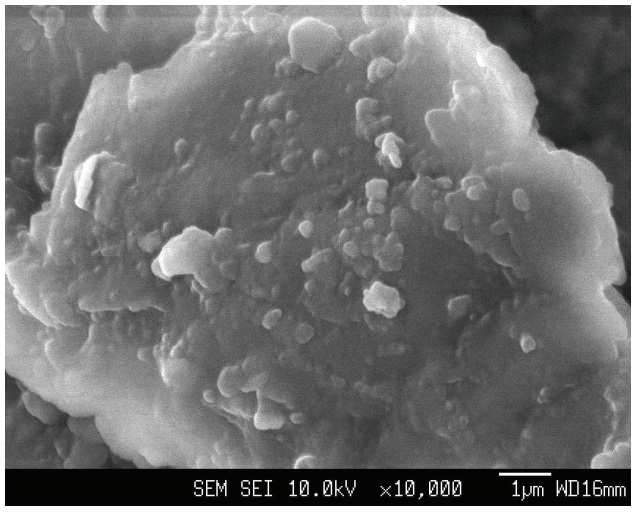

(d)

FIGURE 1: SEM photos of mesoporous silica: $(a)$ the morphology $(\times 1500)$ and $(b)$ the surface $(\times 30000)$ of nonmodified sample, and $(c)$ the morphology $(\times 2000)$ and $(d)$ the surface $(\times 10000)$ of oleic acid modified sample.

FT-IR analysis of samples was performed after heat treatment at various temperatures for $1 \mathrm{~h}$. The spectra of nonmodified original mesoporous material (Figure 4(a)) are similar to that of amorphous silica. Specifically, the bands at $950 \mathrm{~cm}^{-1}, 798 \mathrm{~cm}^{-1}$, and $450 \mathrm{~cm}^{-1}$ are due to Si-OH stretching, $\mathrm{Si}-\mathrm{O}-\mathrm{Si}$ symmetric stretching, and bending vibrations, respectively. The band at $570 \mathrm{~cm}^{-1}$ is due to $\mathrm{Si}-\mathrm{OH}$ vibration $[17,22]$. The sharp band at $1635 \mathrm{~cm}^{-1}$, which is attributed to deformed water that interacts through hydrogen bonds with silanol groups, shows a lower intensity for the samples treated with oleic acid. The peak at $1724 \mathrm{~cm}^{-1}$ corresponding to carbonyl group for adsorbed oleic acid in Figures 4(b)-4(e) indicates that the carbonyl group forms hydrogen bonding with the surface $\mathrm{Si}-\mathrm{OH}$ groups. It is observed that three distinct structures of adsorption of oleic acid onto silica surface coexist as follows: single and double hydrogen bonded chemisorption and physically adsorbed dimeric oleic acid. Pure oleic acid has a carbonyl band at $1713 \mathrm{~cm}^{-1}$ for dimer, so a band for the physically adsorbed dimer on silica could be shown at $1713 \mathrm{~cm}^{-1}$ [23]. A single hydrogen bond of oleic acid molecule adsorbed on silica surface would be expected to have a carbonyl stretch at about $1691 \mathrm{~cm}^{-1}$. The formation of a cyclic double hydrogen bonded dimeric structure would shift the carbonyl frequency up by about $35 \mathrm{~cm}^{-1}$ above that 


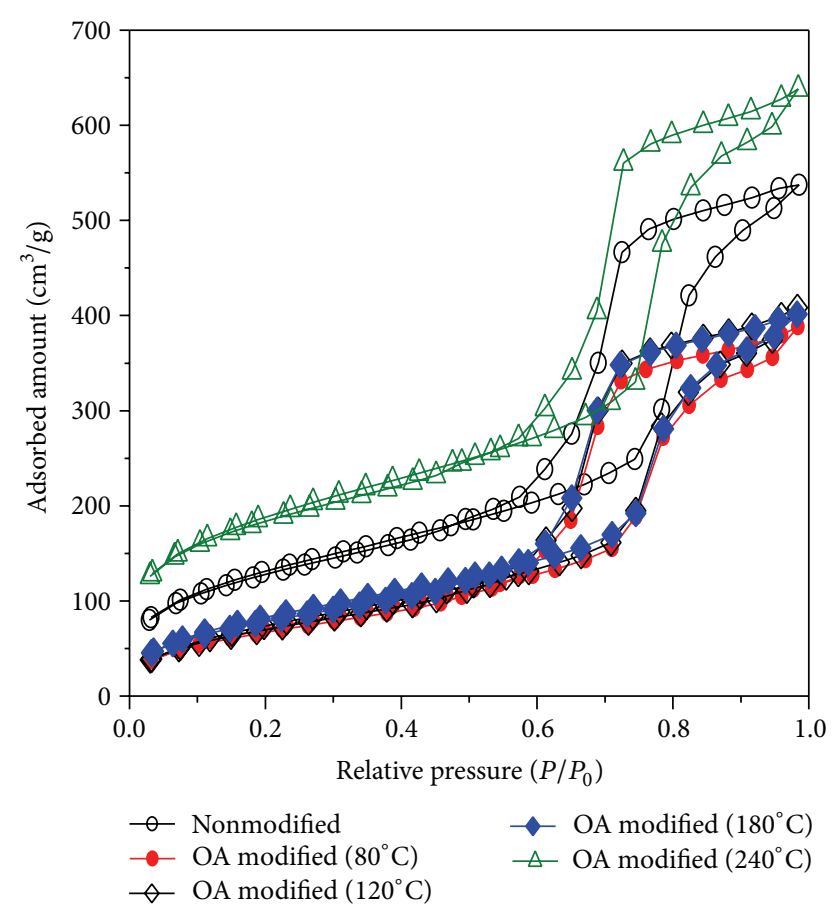

FIGURE 2: Nitrogen adsorption-desorption isotherms of nonmodified sample and oleic acid (OA) modified samples with heat treatment at various temperatures.

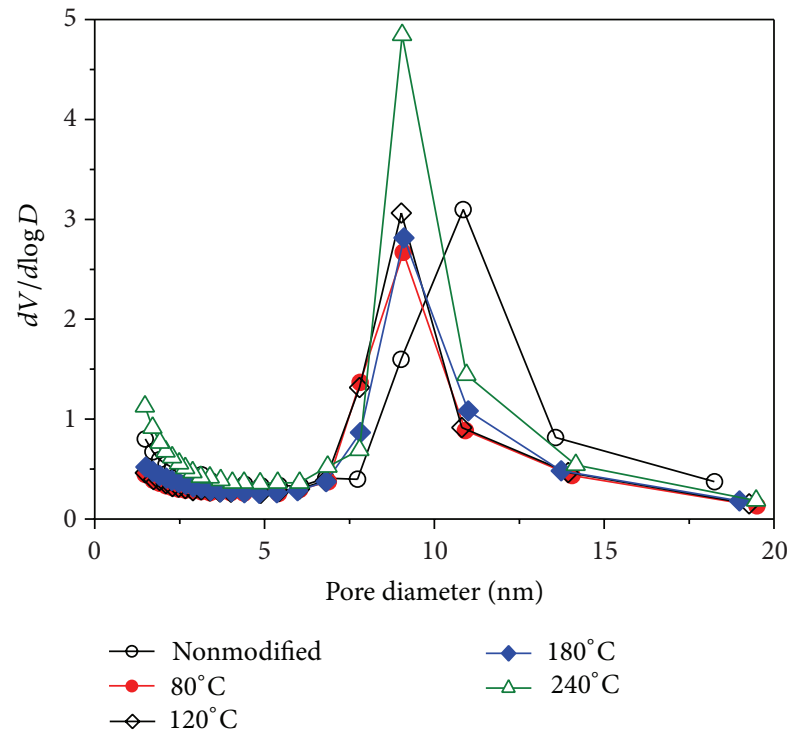

Figure 3: Pore size distributions of nonmodified sample and oleic acid (OA) modified samples with heat treatment at various temperatures.

of the single hydrogen bonded structure [23]. Thus, double hydrogen bonded carbonyl frequency would be expected to be near $1726 \mathrm{~cm}^{-1}$ [23]. In view of the band at $1724 \mathrm{~cm}^{-1}$ in Figures 4(b)-4(e), it is considered that oleic acid is adsorbed on mesoporous silica surface by double hydrogen bond [24]. The broad band at near $3400 \mathrm{~cm}^{-1}$ is associated with

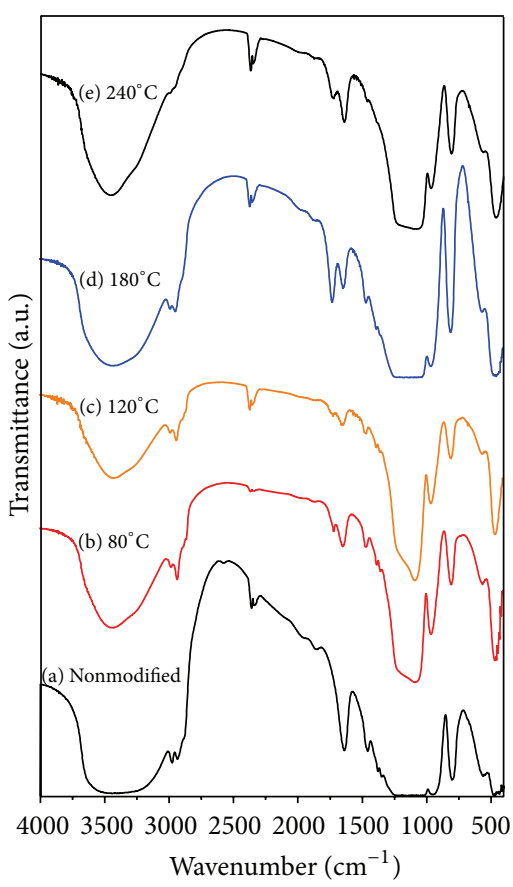

FIGURE 4: FT-IR spectra of nonmodified sample and oleic acid (OA) modified samples with heat treatment at various temperatures.

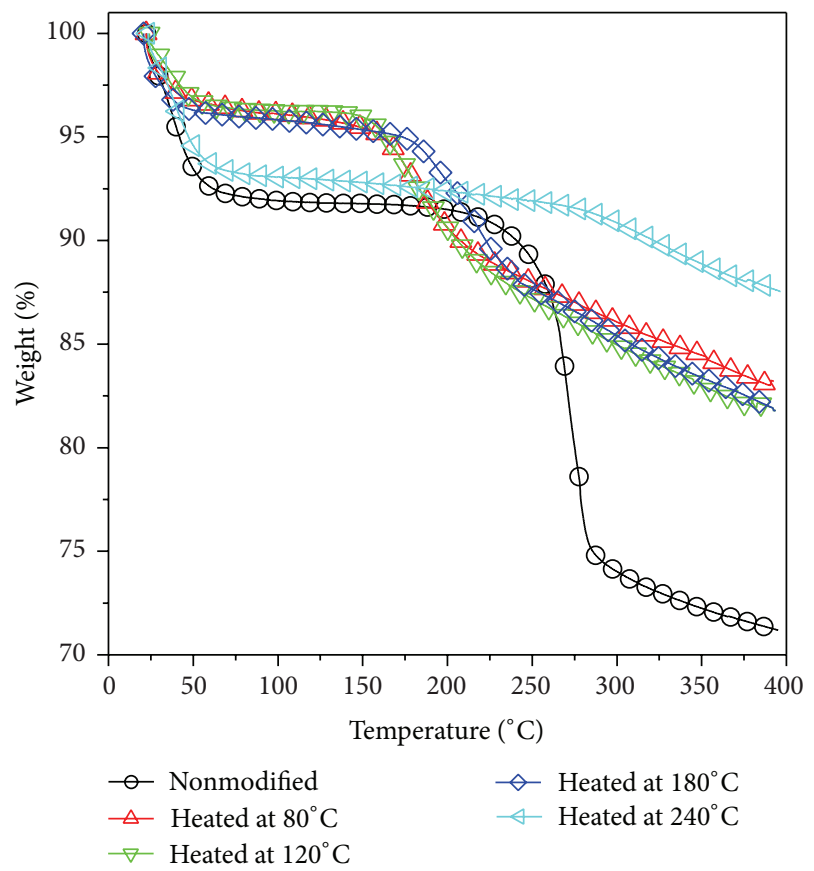

FIGURE 5: TGA curves of nonmodified sample and oleic acid (OA) modified samples with heat treatment at various temperatures.

the silanol stretching of surface and vibrational structure of siloxane by water molecule adsorbed on silica surface. The width of broad bands of oleic acid modified sample is relatively narrow comparing to that of nonmodified sample. It is observed that the intensities of the band of hydroxyl groups 


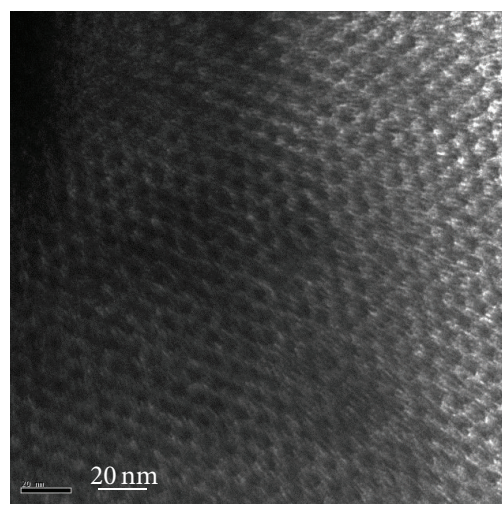

(a)

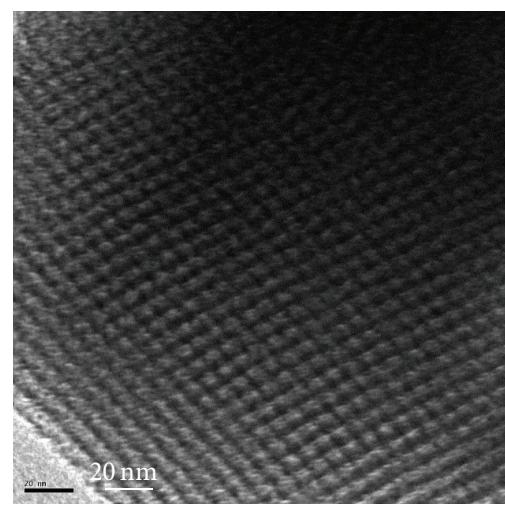

(b)

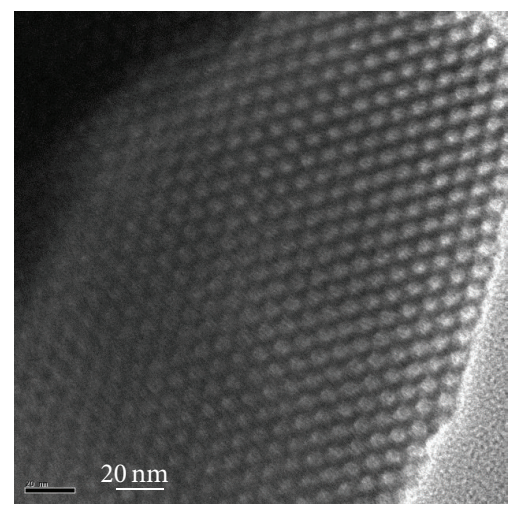

(c)

FIGURE 6: TEM images of nonmodified original sample (a) and oleic acid modified samples with heat treatment at $120^{\circ} \mathrm{C}$ before (b) and after (c) hydrothermal treatment.

between $3250 \mathrm{~cm}^{-1}$ and $3500 \mathrm{~cm}^{-1}$ decreased in the oleic acid modified samples as compared to the original sample. This is due to the hydrophobic characteristic of oleic acid surface, which inhibits significant water adsorption.

3.3. Thermogravimetric Analysis. The thermogravimetric analysis of the samples was carried out for removal of the remained organic surfactant and physically adsorbed oleic acid. Nonmodified original mesoporous material shows rapid weight loss between $230^{\circ} \mathrm{C}$ and $280^{\circ} \mathrm{C}$ (Figure 5) and continuous loss after $280^{\circ} \mathrm{C}$, which is consistent with the observations from previous studies [25-27]. This weight loss may be due to the removal of nonionic surfactant. Though surfactants were washed out with ethanol and water, they were still remained in mesoporous silica [25-27]. Oleic acid modified samples have different trend of weight loss from that of the original material. All the modified samples show lower weight losses than the original silica. The decomposition of oleic acid in modified samples started from $150^{\circ} \mathrm{C}$, and unlike the nonmodified sample the rapid weight loss between $230^{\circ} \mathrm{C}$ and $280^{\circ} \mathrm{C}$ was not observed. The weight loss in the range between ambient temperature and $70^{\circ} \mathrm{C}$ may be due to the desorption of water and some organic substances. In this temperature range, the weight loss of the samples with heat treatment at $120^{\circ} \mathrm{C}$ is smaller than that of the other samples. The trend could be attributed to the less specific surface area of the sample treated at $120^{\circ} \mathrm{C}$ than other materials. However, among the oleic acid treated samples, distinct difference in thermogravimetric analysis was observed for the samples treated at $240^{\circ} \mathrm{C}$. Specifically, the weight loss starting around $150^{\circ} \mathrm{C}$ which is attributed to the decomposition of oleic acid was not observed for the samples treated at $240^{\circ} \mathrm{C}$. It is well known that the thermal decomposition of oleic acid occurs around $200^{\circ} \mathrm{C}$ [28-30]. Hence, the different trend observed in the samples treated at $240^{\circ} \mathrm{C}$ was due to the decomposition of oleic acid by the heat treatment at $240^{\circ} \mathrm{C}$. It is known that the specific surface area of mesoporous silica is mainly affected by micropores formed within the wall of mesopores [31, 32]. Accordingly, higher specific surface area and greater pore volume observed for the samples treated at $240^{\circ} \mathrm{C}$ relative to other oleic acid treated samples are likely due to the development of the micropores in the wall of mesopores via the removal of oleic acid at $240^{\circ} \mathrm{C}$ (Table 1) [33].

3.4. Effects of Oleic Acid on the Stability of Mesoporous Silica. Figure 6 shows TEM images of mesoporous silica prepared in three different ways: nonmodified original sample (Figure 6(a)) and oleic acid treated sample with heat treatment at $120^{\circ} \mathrm{C}$ without (Figure 6(b)) and with (Figure 6(c)) hydrothermal treatment. Overall, distinct changes in mesopore structure were not observed between three samples. Here, it is worthwhile to note that TEM analysis has limitation to evaluate the minute changes in micropore structure [31, 33]. Therefore, in order to further investigate the changes in pore structure nitrogen adsorption-desorption tests were carried out.

When the oleic acid was adsorbed on mesoporous silica, the specific surface area of modified sample reduced about $43 \%$ comparing to that of the original sample. The hydrothermal stability of samples was tested on the one hand in the static water state at $105^{\circ} \mathrm{C}$ and on the other hand in water refluxing state at $25^{\circ} \mathrm{C}$. Table 1 and Figure 7 show the pore characteristics and nitrogen adsorption-desorption isotherms of the oleic acid modified materials treated thermally at various temperatures. For comparison, the isotherms of the nonmodified original material are also presented in Figure 7 (a) and Table 1.

After the hydrothermal treatment and water treatment, the pore volume of the nonmodified original material decreased about $40 \%$ and $23 \%$, respectively. The decrease of the height of hysteresis at $0.6-0.8$ of relative pressure after hydrothermal treatment and water treatment of original materials resulted from the fact that its mesopore volume reduced and pore structure was collapsed and clogged. Such hysteresis also shifts to the left, which means the decrease of pore size. The $\mathrm{N}_{2}$ gas adsorption amount of sample before treatment is greater than that of sample after hydrothermal treatment and water treatment at the range of under 0.3 of relative pressure. Therefore, the hydrothermal treatment and water treatment also caused the decrease of the micropore 


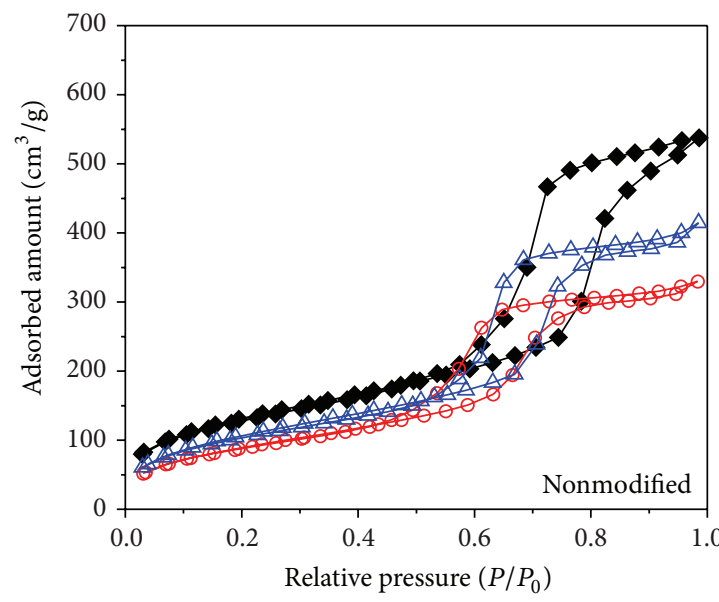

(a)

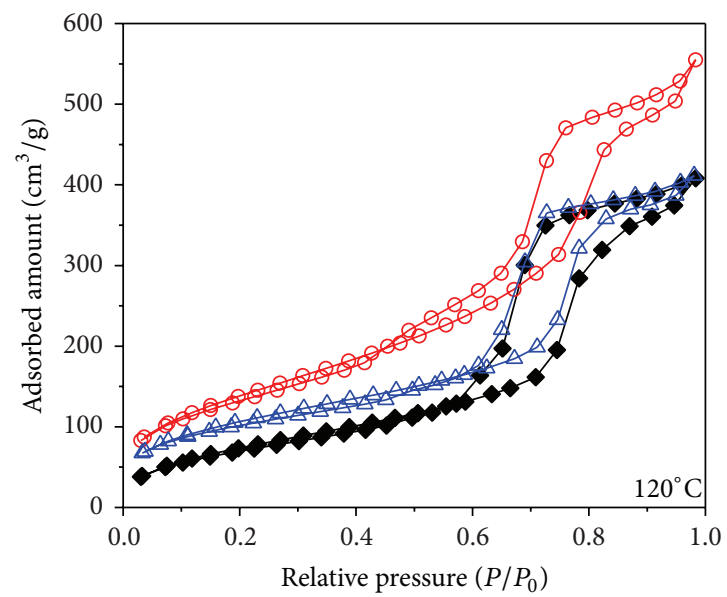

(c)

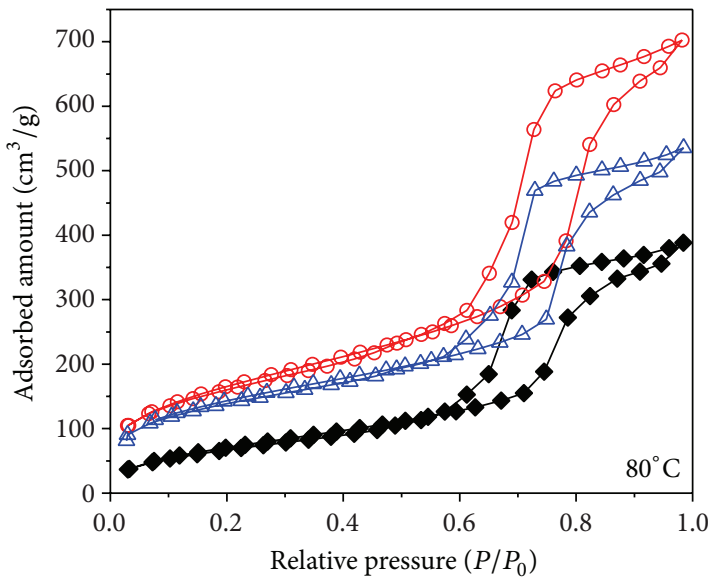

(b)

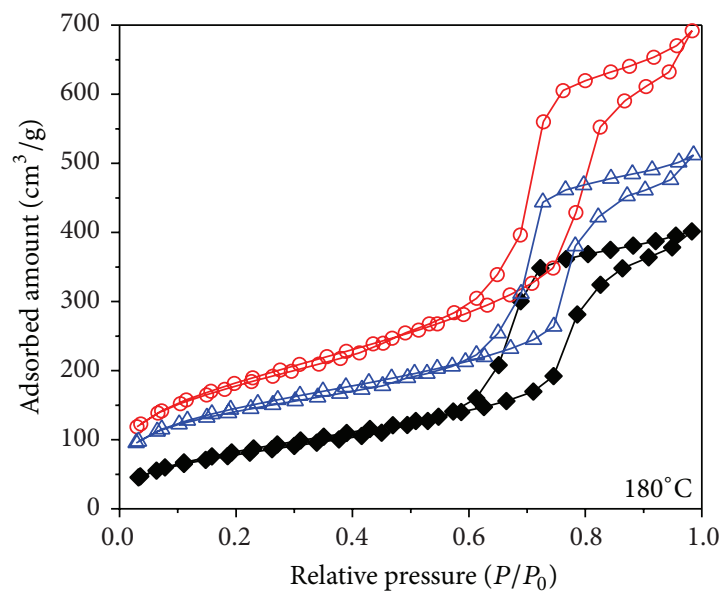

(d)

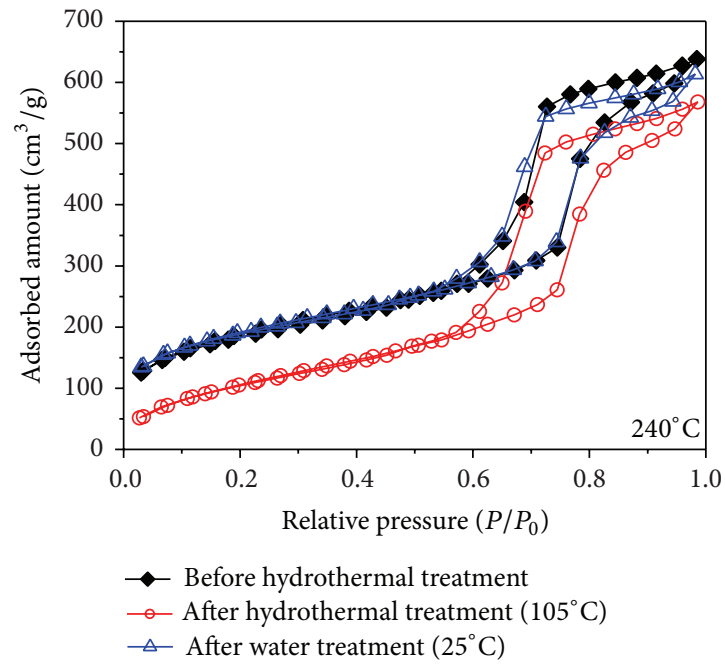

(e)

FIGURE 7: Nitrogen adsorption-desorption of nonmodified sample and oleic acid modified samples with heat treatment at various temperatures before and after hydrothermal and water treatment. 
volume; that is, they were less stable in water state as observed from many previous studies $[2,3]$.

Unlike the nonmodified original material, the mesopore structure of oleic acid modified samples with heat treatment at $80-180^{\circ} \mathrm{C}$ (Figures $7(\mathrm{~b})-7(\mathrm{~d})$ ) was improved after hydrothermal and water treatment comparing to the sample before treatment (Table 1). Specifically, the specific surface areas increased almost two times in hydrothermal treated material at $105^{\circ} \mathrm{C}$ over those of materials before hydrothermal treatment. Similarly, the water treatment at $25^{\circ} \mathrm{C}$ also promotes mesopore structure and pore volume of the oleic acid modified materials, though as less as the case of the hydrothermal treatment. The hydrothermal treatment at $105^{\circ} \mathrm{C}$ may cause the growth of mesopore structure instead of the structure collapse by water invasion, which takes place in nonmodified original mesoporous material. The fact that the combined process of the surface modification process with oleic acid and the hydrothermal/water treatment could improve physical properties of mesoporous materials can be supported by the test results for hydrothermal stability of the oleic acid treated samples with heat treatment at $240^{\circ} \mathrm{C}$. The isotherm of the materials with heat treatment at $240^{\circ} \mathrm{C}$ (Figure $7(\mathrm{e})$ ) shifts to downward after hydrothermal and water treatment, which means the decrease of the pore volume and the specific surface area after hydrothermal treatment. The decrease of specific surface area and the pore volume after hydrothermal treatment and water treatment could be attributed to the decomposition of oleic acid that protects the material surface from water. These results also support that, in this study, the oleic acid adsorbed on the mesoporous silica was thermally stable up to the heat treatment temperature of $180^{\circ} \mathrm{C}$.

Based on the results, the hydrophobic surface modification of mesoporous materials with oleic acid was found to improve their hydrothermal stability, suggesting that more improved mesoporous materials in stability could be synthesized by the hydrophobic surface modification, which protects silica surface against water in more hydrothermal process. Furthermore, the results also indicate that the mesoporous materials with improved physical properties (e.g., specific surface area, pore volume) could be prepared by combining the surface modification process using oleic acid with the hydrothermal treatment.

\section{Conclusions}

The structural analysis of the oleic acid modified samples revealed some reduction in specific surface area, pore volume, and pore diameter because of the high molecular oleic acid adsorption on inner pore surface of silica. The mesopore diameter decreased about $2 \mathrm{~nm}$ by oleic acid modification. It is confirmed that oleic acid treated mesoporous materials were stable thermally up to $180^{\circ} \mathrm{C}$ by heat treatment through FT-IR and thermogravimetric analyses. FT-IR results showed that carbonyl group of oleic acid was bonded strongly with silanol group on silica surface. The hydrophobic surface modification using oleic acid caused the mesoporous material not only to be better stable hydrothermally, but also to obtain more improved mesoporous structure in the hydrothermal process.

\section{Conflict of Interests}

The authors declare that there is no conflict of interests regarding the publication of this paper.

\section{Acknowledgments}

This work was in part supported by the Mine Reclamation Corporation Research Fund and the National Research Foundation of Korea Grant funded by the Korean Government (MEST) (NRF-2011-0014627).

\section{References}

[1] P. Selvam, S. K. Bhatia, and C. G. Sonwane, "Recent advances in processing and characterization of periodic mesoporous MCM-41 silicate molecular sieves," Industrial and Engineering Chemistry Research, vol. 40, no. 15, pp. 3237-3261, 2001.

[2] S. Inagaki and Y. Fukushima, "Adsorption of water vapor and hydrophobicity of ordered mesoporous silica, FSM-16," Microporous and Mesoporous Materials, vol. 21, no. 4-6, pp. 667-672, 1998.

[3] A. Cauvel, D. Brunel, F. Di Renzo, E. Garrone, and B. Fubini, "Hydrophobic and hydrophilic behavior of micelle-templated mesoporous silica," Langmuir, vol. 13, no. 10, pp. 2773-2778, 1997.

[4] K. A. Koyano, T. Tatsumi, Y. Tanaka, and S. Nakata, "Stabilization of mesoporous molecular sieves by trimethylsilylation," Journal of Physical Chemistry B, vol. 101, no. 46, pp. 9436-9440, 1997.

[5] K. Yamamoto and T. Tatsumi, "Remarkable improvement in hydrothermal stability of MCM-41 by surface modification with Grignard reagents," Chemistry Letters, no. 6, pp. 624-625, 2000.

[6] K. R. Kloetstra, M. van Laren, and H. van Bekkum, "Binary caesium-lanthanum oxide supported on MCM-41: A new stable heterogeneous basic catalyst," Journal of the Chemical SocietyFaraday Transactions, vol. 93, no. 6, pp. 1211-1220, 1997.

[7] L. Huang, W. Guo, P. Deng, Z. Xue, and Q. Li, "Investigation of synthesizing MCM-41/ZSM-5 composites," The Journal of Physical Chemistry B, vol. 104, no. 13, pp. 2817-2823, 2000.

[8] K. R. Kloetstra, H. W. Zandbergen, J. C. Jansen, and H. Van Bekkum, "Overgrowth of mesoporous MCM-41 on faujasite," Microporous Materials, vol. 6, no. 5-6, pp. 287-293, 1996.

[9] A. Karlsson, M. Stöcker, and R. Schmidt, "Composites of micro- and mesoporous materials: simultaneous syntheses of MFI/MCM-41 like phases by a mixed template approach," Microporous and Mesoporous Materials, vol. 27, no. 2-3, pp. 181$192,1999$.

[10] A. Hidrobo, J. Retuert, P. Araya, and E. Wolf, "Stable zeolitecontaining mesoporous aluminosilicates," Journal of Porous Materials, vol. 10, no. 4, pp. 231-234, 2003.

[11] M. C. Capel-Sanchez, L. Barrio, J. M. Campos-Martin, and J. L. G. Fierro, "Silylation and surface properties of chemically grafted hydrophobic silica," Journal of Colloid and Interface Science, vol. 277, no. 1, pp. 146-153, 2004.

[12] S. Jung and H. Park, "Control of surface residual -OH polar bonds in $\mathrm{SiO}_{2}$ aerogel film by silylation," Thin Solid Films, vol. 420-421, pp. 503-507, 2002. 
[13] K. Yamamoto and T. Tatsumi, "Organic functionalization of mesoporous molecular sieves with Grignard reagents," Microporous and Mesoporous Materials, vol. 44-45, pp. 459-464, 2001.

[14] P. K. Jal, S. Patel, and B. K. Mishra, "Chemical modification of silica surface by immobilization of functional groups for extractive concentration of metal ions," Talanta, vol. 62, no. 5, pp. 1005-1028, 2004.

[15] C. F. Nie, R. Zhao, and J. S. Suo, "Synthesis and characterization of bifunctional periodic silica with surface and framework benzene functionality," Journal of Porous Materials, vol. 11, no. 3, pp. 141-146, 2004.

[16] Z. W. Li and Y. F. Zhu, "Surface-modification of $\mathrm{SiO}_{2}$ nanoparticles with oleic acid," Applied Surface Science, vol. 211, no. 1-4, pp. 315-320, 2003.

[17] Y. Han, H. Kim, J. Park, S. Lee, and J. Kim, "Influence of Ti doping level on hydrogen adsorption of mesoporous Ti-SBA15 materials prepared by direct synthesis," International Journal of Hydrogen Energy, vol. 37, no. 19, pp. 14240-14247, 2012.

[18] J. Park, J. Park, and H. Shin, “The preparation of Ag/mesoporous silica by direct silver reduction and $\mathrm{Ag} /$ functionalized mesoporous silica by in situ formation of adsorbed silver," Materials Letters, vol. 61, no. 1, pp. 156-159, 2007.

[19] J. Yu, S. Liu, and H. Yu, "Microstructures and photoactivity of mesoporous anatase hollow microspheres fabricated by fluoride-mediated self-transformation," Journal of Catalysis, vol. 249, no. 1, pp. 59-66, 2007.

[20] J. Yu, J. C. Yu, M. K.-P. Leung et al., "Effects of acidic and basic hydrolysis catalysts on the photocatalytic activity and microstructures of bimodal mesoporous titania," Journal of Catalysis, vol. 217, no. 1, pp. 69-78, 2003.

[21] K. S. W. Sing, D. H. Everett, R. A. W. Haul et al., "Reporting physisorption data for gas solid systems with special reference to the determination of surface-area and porosity (recommendations 1984)," Pure and Applied Chemistry, vol. 57, no. 4, pp. 603-619, 1984.

[22] H. Jeon, S. Yi, and S. Oh, "Preparation and antibacterial effects of $\mathrm{Ag}-\mathrm{SiO}_{2}$ thin films by sol-gel method," Biomaterials, vol. 24, no. 27 , pp. 4921-4928, 2003.

[23] G. Blyholder, C. Adhikar, and A. Proctor, "Structure and orientation of oleic acid adsorbed onto silica gel," Colloids and Surfaces A: Physicochemical and Engineering Aspects, vol. 105, no. 1, pp. 151-158, 1995.

[24] K. Marshall and C. H. Rochester, "Infrared study of the adsorption of oleic and linolenic acids onto the surface of silica immersed in carbon tetrachloride," Journal of the Chemical Society 1: Physical Chemistry in Condensed Phases, vol. 71, pp. 1754-1761, 1975.

[25] D. Solís, E. Vigueras-Santiago, S. Hernández-López, A. GómezCortés, M. Aguilar-Franco, and M. A. Camacho-López, "Textural, structural and electrical properties of $\mathrm{TiO}_{2}$ nanoparticles using Brij 35 and P123 as surfactants," Science and Technology of Advanced Materials, vol. 9, no. 2, Article ID 025003, 2008.

[26] C. Peng, H. Zhang, J. Yu et al., "Synthesis, characterization, and luminescence properties of the ternary europium complex covalently bonded to mesoporous SBA-15," The Journal of Physical Chemistry B, vol. 109, no. 32, pp. 15278-15287, 2005.

[27] N. Xiao, L. Wang, S. Liu et al., "High-temperature synthesis of ordered mesoporous silicas from solo hydrocarbon surfactants and understanding of their synthetic mechanisms," Journal of Materials Chemistry, vol. 19, no. 5, pp. 661-665, 2009.

[28] M. V. Limaye, S. B. Singh, S. K. Date et al., "High coercivity of oleic acid capped $\mathrm{CoFe}_{2} \mathrm{O}_{4}$ nanoparticles at room temperature,"
The Journal of Physical Chemistry B, vol. 113, no. 27, pp. 90709076, 2009.

[29] L. Zhang, R. He, and H.-C. Gu, "Oleic acid coating on the monodisperse magnetite nanoparticles," Applied Surface Science, vol. 253, no. 5, pp. 2611-2617, 2006.

[30] S. Ayyappan, G. Panneerselvam, M. P. Antony et al., "Effect of initial particle size on phase transformation temperature of surfactant capped $\mathrm{Fe}_{3} \mathrm{O}_{4}$ nanoparticles," Journal of Applied Physics, vol. 109, no. 8, Article ID 084303, 2011.

[31] J. Zhu, K. Kailasam, X. Xie, R. Schomaecker, and A. Thomas, "High-surface-area SBA-15 with enhanced mesopore connectivity by the addition of poly(vinyl alcohol)," Chemistry of Materials, vol. 23, no. 8, pp. 2062-2067, 2011.

[32] A. Silvestre-Albero, E. O. Jardim, E. Bruijn et al., "Is there any microporosity in ordered mesoporous silicas?" Langmuir, vol. 25, no. 2, pp. 939-943, 2009.

[33] Y. Han, J. Choi, M. Tong, and H. Kim, "Synthesis and characterization of high-surface-areamillimeter-sized silica beads with hierarchical multi-modal pore structure by the addition of agar," Materials Characterization, vol. 90, pp. 31-39, 2014. 

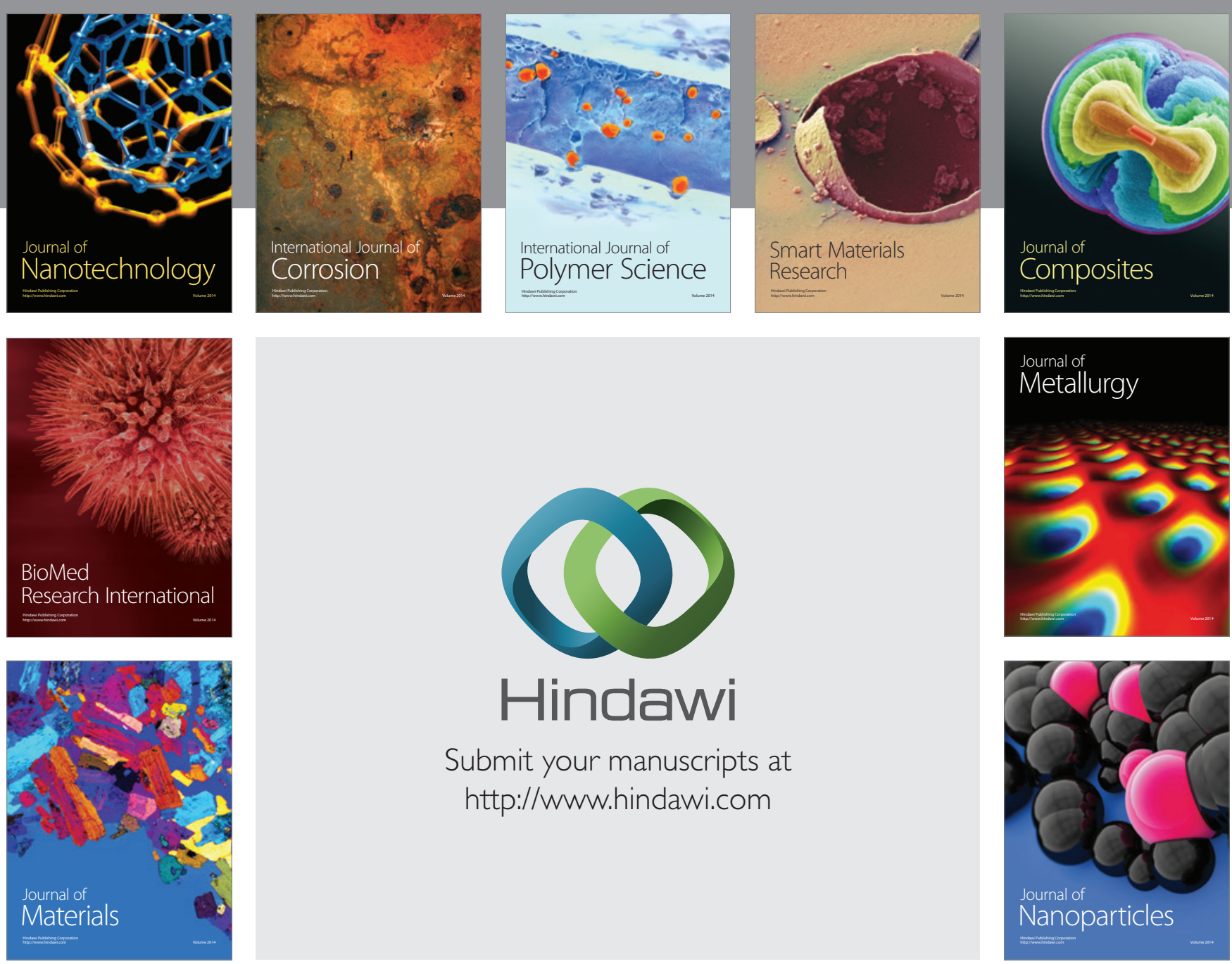

Submit your manuscripts at http://www.hindawi.com
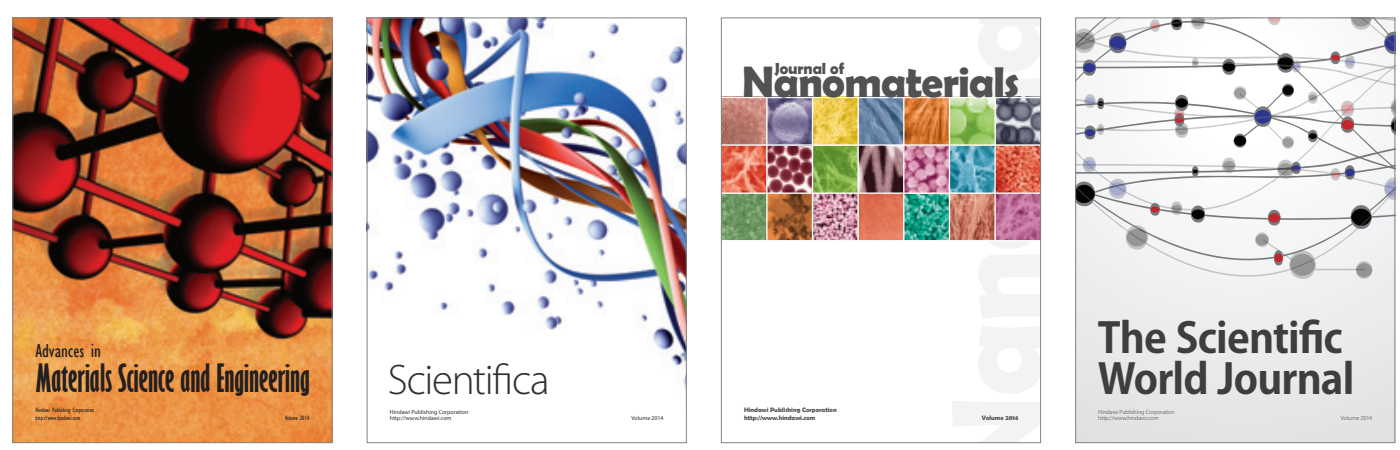

\section{The Scientific World Journal}
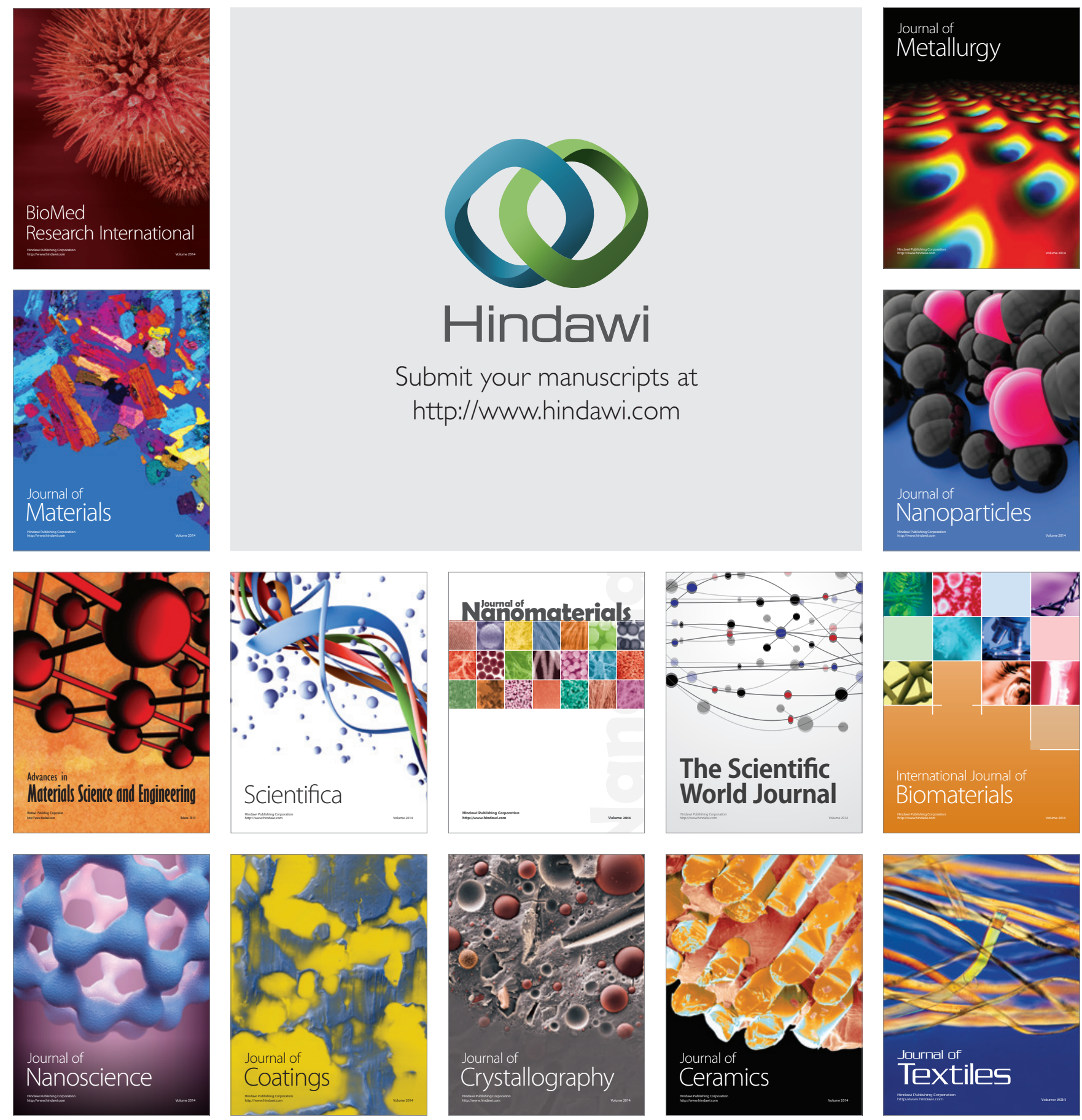